Revue européenne des sciences sociales

European Journal of Social Sciences

XLIV-134 | 2006

Quel(s) défi(s) pour les sciences sociales à l'heure de la mondialisation?

\title{
Eugène Dupréel, philosophe, sociologue et moraliste
}

Jacques Coenen-Huther

\section{CpenEdition}

Journals

Édition électronique

URL : http://journals.openedition.org/ress/288

DOI : $10.4000 /$ ress. 288

ISSN : 1663-4446

Éditeur

Librairie Droz

Édition imprimée

Date de publication : 1 juin 2006

Pagination : $97-118$

ISBN : 9-782-600-01095-5

ISSN : 0048-8046

Référence électronique

Jacques Coenen-Huther, "Eugène Dupréel, philosophe, sociologue et moraliste », Revue européenne des sciences sociales [En ligne], XLIV-134 | 2006, mis en ligne le 14 octobre 2009, consulté le 19 avril 2019. URL : http://journals.openedition.org/ress/288 ; DOI : 10.4000/ress.288

(c) Librairie Droz 


\section{EUGÈNE DUPRÉEL, PHILOSOPHE, SOCIOLOGUE ET MORALISTE}

Eugène Dupréel (1879-1967) fut professeur à l'Université Libre de Bruxelles de 1906 à 1949. Elu membre correspondant de l'Académie Royale de Belgique en 1927, il en devint membre titulaire en 1939. Il fut également membre correspondant de l'Institut de France et docteur honoris causa de la Sorbonne. Après une formation initiale en histoire, il s'orienta vers la philosophie sous l'influence de René Berthelot qui enseignait alors à Bruxelles et il devint le plus original des philosophes belges de son temps. Il développa également la sociologie d'une société complexe, refusant les extrêmes du holisme et de l'individualisme. Cette sociologie est redevable aux influences conjuguées de Simmel et de Tarde mais n'en refuse pas pour autant l'apport durkheimien. Ce qui frappe les familiers de l'œuvre de Dupréel, c'est une grande cohérence conférant une unité d'ensemble aux conceptions philosophiques, sociologiques et morales de son auteur. La sociologie de Dupréel, on le verra, se situe dans le prolongement de sa philosophie qui lui imprime son orientation pluraliste générale. Dupréel était d'ailleurs d'avis que «le sociologue non philosophe serait un peu comme ces astronomes amateurs qui ignorent les mathématiques: il y a dans les phénomènes qu'ils étudient des enchaînements qui leur échappent, et dans les représentations qu'ils s'en font, des lacunes qu'ils leur faut combler par des affirmations reçues d'autrui et qu'ils ne sauraient contrôler» (1932, p. 282). Mais cette philosophie dont Georges Bastide dira qu'elle offre «le spectacle inattendu d'une recherche originale, irréductible à des classifications préformées » $(1968$, p. 41) doit une partie de son originalité au soubassement sociologique dont elle bénéficie et qui lui apporte la notion de convention, génératrice de réalité tout comme de dynamique sociale. Quant aux conceptions de Dupréel en matière de morale, elles trouvent leur fondement dans des réflexions philosophiques et sociologiques. La moralité est un fait social, ce qui revient à dire qu'elle est « inexplicable en dehors des relations entre plusieurs individus (Dupréel, 1932, p. 220). La démarche morale ne relève pas « d'une seule et même conscience individuelle»; il faut y voir au contraire « un complexe d'activités, qui ne saurait tenir tout entier dans le cadre d'une seule conscience» (1932, p. 170). Le mérite moral repose certes sur l'une ou l'autre forme de renoncement, c'est-à-dire sur l'acceptation d'un détriment, mais aussi sur le respect d'une règle approuvée socialement.

Il n'est pas exagéré d'affirmer qu'Eugène Dupréel exerça, bien au-delà de son accession à l'éméritat, une influence considérable sur plusieurs générations d'intellectuels bruxellois alors qu'il avait acquis une stature internationale incontestable. Depuis la fin des années 1960, le rayonnement de son œuvre a néanmoins 
pâli considérablement, au point que des sociologues de la jeune génération ignorent jusqu'à son nom. Qu'il puisse difficilement être rattaché à une école ou à un courant de pensée organisé et qu'il se soit lui-même peu soucié de faire école contribue sans doute largement à cet effacement. On espère pourtant montrer ici que ses travaux sont riches d'enseignements et restent une source d'inspiration pour les praticiens des sciences sociales.

\section{DU REFUS DE LA CONNAISSANCE NÉCESSAIRE AU PROBABILISME}

La clé de voûte de la philosophie de Dupréel est le rejet de l'idée de connaissance nécessaire. Cette prise de position philosophique est audacieuse si l'on songe que l'idée de connaissance nécessaire a joué un rôle éminent dans la philosophie classique et qu'elle reste une aspiration de l'esprit scientifique. Il vaut la peine d'examiner en détail le raisonnement qui conduit à ce rejet. La nécessité attribuée à une connaissance vise à sélectionner des propositions qu'on ne peut pas ne pas tenir pour vraies. Ce critère, estime Dupréel, est cependant loin d'être infaillible: la vérité nécessaire n'est rien d'autre que celle qui nous paraît nécessaire, compte tenu de l'état général de nos connaissances. Il apparaissait «aussi nécessaire» aux Anciens «que la terre fût plate ou qu'il y eût dans l'univers un haut et un bas qu'il a paru inévitable au physicien classique que tout phénomène eût une cause» (Dupréel, 1928b, 1949, p. 76). La nécessité philosophique se ramène ainsi à une intuition: celle de l'absurdité de la proposition contraire. Sur ce point, Dupréel s'oppose clairement à toutes les philosophies intuitionnistes. L'intuition ne peut être ni vérifiée ni partagée et pour tous ceux qui n'en ont pas été saisis, elle «n'est qu'un fait» dont on ne peut que prendre acte. C'est ainsi la logique qui s'efface devant «une sorte d'expérience psychologique». Dès lors, on ne peut que tenir la nécessité pour une notion équivoque. Elle ne saurait être confondue avec l'évidence. Celle-ci exprime un état de la conscience individuelle et peut comporter des degrés. On peut en effet considérer une proposition comme plus ou moins évidente alors «qu'il serait contradictoire de parler d'une affirmation plus ou moins nécessaire» (ibid., p. 79). Dans le cas d'un raisonnement ou d'un calcul long et compliqué, il peut se faire qu'un sentiment de nécessité s'impose à l'esprit de façon identique au point de départ et à la conclusion. L'évidence, au contraire, «fléchit d'autant plus que les opérations partielles sont plus longues et plus nombreuses » (ibid., p. 83) ${ }^{1}$. Il n'y a donc pas de parallélisme entre nécessité et évidence. On ne peut pourtant pas non plus fonder la notion de connaissance nécessaire sur celle de simplicité. Il faudrait pour cela qu'on puisse identifier à coup sûr une connaissance simple. Mais une fois de plus, on ne peut que se fier à une intuition: «soit l'intuition directe d'une unité indivisible, soit plutôt l'intuition de l'incapacité où nous sommes de pousser plus loin l'analyse»

\footnotetext{
Dupréel illustre ceci par un exemple simple: soit la formule $a+b=c$. Si on lui donne la valeur $2+2=4$, «évidence et nécessité paraissent confondues dans une seule et même intuition». Si en revanche on substitue aux termes $a+b$, des nombres très grands ou des quantités très complexes, «la dissociation de l'évidence et de la nécessité se produit» (1928b, 1949, p. 84).
} 
(ibid., p. 82). Ainsi l'intuition - voire même l'intuition de carence - est le moment obligé de l'établissement du jugement nécessaire. Pour Dupréel, ce rôle essentiel de l'intuition ne peut qu'être ressenti comme un échec de la pensée rationnelle qui entend fonder ses démarches sur le raisonnement et la logique. C'est alors le rationalisme qui le cède à l'intuitionnisme. Si la pensée classique s'appuie sur la notion de vérité nécessaire c'est parce qu'elle ne peut concevoir le progrès de la connaissance qu'à partir d'un point fixe, solidement établi. Or, estime Dupréel, si des vérités s'imposent à nous de façon immédiate, «par la seule intuition de notre impuissance à en éviter l'affirmation», c'est bien le signe «qu'elles expriment, non notre savoir, mais notre ignorance» (ibid., p. 91; souligné par l'auteur). Il n'y a aucune raison de considérer le cheminement de la pensée humaine comme un «voyage dans une seule direction», à partir d'un point de départ qui ne doive jamais être remis en question. Les progrès de nos connaissances élargissent le champ que maîtrise notre pensée «entre deux inconnus » (ibid., p. 92): en amont, l'inconnu que masquent les jugements réputés nécessaires parce qu'ils se dérobent à toute analyse; en aval, l'inconnu des domaines encore à explorer et à comprendre.

Pour que la notion de vérité nécessaire puisse être l'élément premier, conférant un caractère incontestable à tout raisonnement qui la prenne comme point de départ, il faudrait qu'on puisse y voir une sorte d'atome logique insécable. C'est bien ainsi qu'elle apparaît chez de nombreux auteurs qui tendent à confondre dans la catégorie de «l'être » les propriétés de deux éléments qu'il importe au contraire selon Dupréel de dissocier: l'ordre, au sens logique d'espèce ou de catégorie, et la force, au sens de principe d'action à même de créer une dynamique (ibid., p. 85-87). Car la notion de vérité nécessaire est bel et bien une notion composite, résultant de la conjonction instable d'un principe logique et d'un principe dynamique. Accorde-t-on le primat à la notion logique d'ordre, on tombe dans le déterminisme absolu. Donne-t-on au contraire la primauté au principe actif de la force, on est livré à la contingence pure (1939, p. 47-48). Entre ces deux extrêmes du déterminisme et de la contingence, il y a place pour une conception plus subtile: celle de la convergence limitée de deux éléments qui restent hétérogènes l'un à l'autre. On écarte ainsi l'idée de nécessité philosophique au profit de la notion de probabilité. Un jugement n'est jamais rigoureusement nécessaire car un principe actif peut à tout moment bouleverser un ordre logique. La conséquence d'une action n'est jamais que probable car des phénomènes intercalaires viennent inévitablement s'insérer dans la suite logique des antécédents et des conséquents (1939, p. 48-49); c'est ce que Gurvitch, se référant à Dupréel, qualifie d'élément de «perturbation» intervenant entre la cause et l'effet (Gurvitch, 1955, p. 42).

Pour ce qui est de la détermination des faits et de leur enchaînement, la logique probabiliste ne constitue nullement un recul par rapport à la logique causale et Dupréel le montre par un exemple simple. Lorsqu'on prévoit le passage d'un homme entre deux points $\mathrm{A}$ et $\mathrm{B}$, cette prévision peut se fonder sur deux types d'informations. Il peut s'agir d'une détermination précise de l'agent: on dispose de données concernant un individu particulier et les intentions qu'il manifeste. Il peut s'agir au contraire d'une connaissance suffisante de l'intervalle A-B: il s'y trouve un point de passage obligé et un individu au moins ne peut qu'y passer. La 
première démarche correspond à une logique causale, la seconde à une logique probabiliste (1933-1934, 1949, p. 236-237). Pour Dupréel, la détermination par la probabilité permet de «serrer le réel» d'aussi près qu'une démarche causale ${ }^{2}$. Si l'on considère le rapport d'un effet à sa cause, la valeur opératoire de la notion de cause réside dans la discontinuité qu'elle établit entre un phénomène «cause » et un phénomène «effet $»^{3}$. L'idée d'un rapport de cause à effet n'a de sens qu'en présence de deux phénomènes distincts. Qu'ils soient distincts implique l'existence entre eux d'un intervalle, qu'il soit infime ou considérable. La réalité intercalaire - les événements qui se produisent dans l'intervalle - rend compte de l'effet au même titre que la cause initiale. Supposer que les événements intercalaires ne viennent que renforcer l'impact de la cause revient à ériger en règle générale ce qui n'est qu'un cas particulier: celui où l'intervalle ne comporte que des faits favorables à ce renforcement. La cause étant donnée, la production de l'effet n'est donc que probable. Ce que le sens commun et la philosophie classique considèrent comme relevant de la causalité se produit en fait dans un «cadre de probabilité» dont la cause n'est qu'un élément (1933, 1949, p. 199-202).

\section{DU PROBABILISME AU PLURALISME ORDINAL}

Le cadre de probabilité est un ordre, c'est-à-dire une catégorie statique, au sein duquel intervient un principe actif: un ou plusieurs événements se produisant dans l'espace intercalaire entre cause et effet. C'est le rapport entre ordre et événement qui permet de supputer une éventualité. Lorsque le cadre de probabilité est complètement circonscrit par les particularités de l'agent, son pouvoir prédicteur sur l'événement est très fort: une éventualité s'impose comme la plus probable. Mais l'intervention d'un principe actif extérieur n'est jamais à exclure. Le rôle d'un tel élément intercalaire peut s'avérer décisif: «c'est alors l'imprévu qui arrive» (1933-1934, 1949, p. 240). Entre déterminisme et contingence, la réalité résulte toujours de la combinaison d'un ordre avec «de l'acte ou de la force» venant de l'extérieur de cet ordre. Car sans intervention extérieure, l'ordre ne pourrait être «énoncé ni discerné » (1939, p. 47-48). L'intervention extérieure émane d'un autre ordre préalablement adopté. Tenter d'imaginer un ordre unique reviendrait à dissoudre la notion d'ordre: une espèce ou une catégorie ne peut exister qu'en se distinguant d'une autre espèce ou d'une autre catégorie. Ceci est un principe de portée tout à fait générale, vraisemblablement hérité de l'hegelianisme de René Berthelot ${ }^{4}$ et qui domine la pensée de Dupréel. Celui-ci l'applique

\footnotetext{
2 Gurvitch, s'appuyant sur Dupréel, qualifie le calcul des probabilités de «technique de réconciliation particulièrement poussée entre cohérence et contingence» (1955, p. 51).

Précisons que Dupréel opère une distinction entre cause «au sens large, ou relatif», à savoir «tout antécédent quelconque qui ... conditionne à quel titre que ce soit l'existence ou la manière d'être » d'un conséquent, et cause « au sens étroit, ou absolu » (1933, 1949, p. 198). C'est à cette causalité au sens restreint qu'il oppose la logique probabiliste.

4 Pour R. Berthelot, «la notion d'identité pure et simple, abstraite de toute idée de différence, est inconcevable» (1908, p. 182).
} 
à la vie sociale tout comme à l'ordre des valeurs. Il en résulte une orientation pluraliste, tant en sociologie qu'en philosophie. Tout ordre repose sur un choix mais tout choix émane d'un ordre: il existe une pluralité d'ordres qui se définissent mutuellement. Les ordres étant relatifs et multiples, ils impliquent inévitablement un élément dynamique: tout ce qui relève du changement. Dès qu'il y a «différence ou distance logique», il y a action (ibid., p. 60). Ordre et force s'appellent donc de manière irrésistible. Ils ne peuvent néanmoins subsister qu'indépendamment l'un de l'autre. Il ne peut y avoir de primat. Poser le primat de l'acte serait attribuer à l'acte les qualités de l'ordre; poser au contraire le primat de l'ordre et « voir dans le logique pur l'essence même des choses » serait incorporer dans les propriétés de l'ordre, les caractéristiques de l'action. La dualité irréductible de l'ordre et de l'acte - ou de la force - constitue « une sorte de plafond logique» qui doit être compris, philosophiquement, comme «le point terminus de l'intelligibilité» (ibid., p. 64). La notion de plafond logique est au fondement de la pluralité des ordres et sera la base du pluralisme ordinal de Dupréel. Cette pluralité est à la fois logique et sociologique. Elle apparaît très clairement dans l'analyse que présente Dupréel des causes du rire (1928a, 1949, p. 27-69). Pour lui, l'insuffisance de la causalité classique se manifeste «dans les explications traditionnelles du rire» $(1933,1949$, p. 217 et ss.). La démarche qui consiste à tenter d'en identifier "une cause générale» est vouée à l'échec. La cause n'est jamais aussi générale et aussi exclusive qu'on aurait pu l'imaginer: elle «ne rend jamais le rire assez probable»; son absence «ne rend jamais le rire assez improbable». Une explication adéquate ne peut se fonder sur «telle ou telle cause» qui agirait avec certitude mais bien sur «les circonstances d'ordre social qui rendent le rire infiniment probable» et qui constituent son cadre de probabilité. L'explication ne peut surgir que de l'irruption d'éléments contingents - « le fortuit, le survenant, l'infinité des accidents de toutes sortes» - au sein du cadre de probabilité.

\section{DU PLURALISME ORDINAL AU PLURALISME SOCIOLOGIQUE}

Au cœur du pluralisme de Dupréel, il y a la notion de convention qui établit les passerelles entre philosophie, sociologie et conceptions morales. La convention est opposée à toute vérité nécessaire qui s'imposerait de façon contraignante (Perelman, 1968, p. 228). C'est un fait social, inséparable de la pluralité des groupes et des affiliations, mais c'est aussi une exigence fonctionnelle de la coexistence des groupes et des intérêts divers. Elle implique le compromis entre les conceptions des parties en présence et n'est viable que moyennant des sacrifices consentis; en cela, elle constitue «l'acte spirituel par excellence» (Dupréel, 1925, 1949, p. 13), l'acte par lequel s'instaure la morale (Croissant, 1968, p. 208). La convention "s'insère sur une réalité qui lui est préalable et qu'elle n'a pas créée». Ses antécédents de toutes sortes aboutissent à des «raisons d'agir» convergeant vers le «consentement collectif» qui lui donne forme. Les raisons qui emportent le consentement peuvent être très diverses. Les auteurs de la convention peuvent être mus par «des causes différentes et variables» et leurs motifs peuvent être à leur tour «différents et variables». C'est le «principe des raisons diverses » auquel Dupréel accorde une importance décisive (1925, 1949, 
p. 7). Selon lui, il fut trop souvent négligé. Des penseurs comme Hume, Adam Smith ou Bentham ont certes envisagé « un vaste système de conventions » mais celui-ci était subordonné à l'élément unificateur de l'intérêt général. Quant à Poincaré, s'il se réfère à la notion de convention, c'est pour y voir un «parallélisme des volontés » déterminées par des causes identiques (ibid., p. 8-9). C'est pourtant la diversité des raisons et des préoccupations sous-jacentes qui confère à la convention une indépendance relative «à l'égard de l'une quelconque de ses causes, fins ou raisons d'être» (ibid., p. 10). Au désordre et à l'incohérence, la convention substitue la règle communément acceptée. Elle établit ainsi une réalité nouvelle par «le système de formes » qu'elle met en place. Et c'est dans sa forme qu'elle trouve sa valeur. La convention est génératrice de réalité objective et subjective: si une manière de procéder apparaît comme conventionnelle, c'est parce qu'on a pris conscience de la possibilité d'agir autrement. «Si l'espace euclidien s'avère conventionnel, c'est parce que les mathématiciens ont aperçu la possibilité de faire de la géométrie indépendamment du postulat d'Euclide» (ibid., p. 11). L'esprit de la convention introduit incontestablement dans les rapports humains une certaine dose de relativisme. Un ordre manifeste sa cohérence en se distinguant d'un autre ordre; une valeur s'affirme par opposition à une autre valeur.

Le conventionnalisme est le plus souvent associé aux philosophies antirationalistes et intuitionnistes. Dupréel en est conscient mais il ne juge pas indispensable qu'il en soit ainsi. Le conventionnalisme qu'il propose est pour lui un rationalisme «plus pur et plus systématique » que les doctrines attachées au primat du réel sur le conventionnel. Cette volonté d'envisager simultanément la «consistance du réel» et la «précarité du conventionnel» apparaît dans son attitude à l'égard de la philosophie kantienne. La philosophie de Kant peut selon lui se définir comme un formalisme, mais c'est un formalisme qui n'associe nullement la notion de convention à celle de forme. Les formes a priori de l'entendement sont pour Kant des formes «universelles et nécessaires ». Le formalisme tel que l'entend Dupréel substitue au formalisme unitaire de Kant un formalisme «multiple et indéterminé». Et cet aménagement du formalisme se fait au moyen «de l'usage réitéré de la notion de convention» $(1925,1949$, p. 15). Le progrès post-kantien, selon Dupréel, consiste à cesser d'aborder les problèmes de connaissance et de moralité "par la considération d'un seul esprit ou d'une seule conscience » et à envisager au contraire « une pluralité d'esprits en relation les uns avec les autres» afin d'aboutir à une «philosophie des esprits multiples» (ibid. p. 16-17). On peut objecter à cette position philosophique qu'il n'est pas possible d'établir «le canevas des rapports entre les esprits » si on n'a pas posé au préalable «chacun de ces esprits». Cependant, Dupréel n'accepte sur ce point aucune relation unidirectionnelle. Certes, «il faut des briques pour en faire un mur. Mais c'est parce qu'il y a des murs qu'il y a des briques ». La convention implique des êtres conscients; lorsqu'une convention est «instituée et respectée», on peut en conclure à l'existence d'êtres «spirituels, intelligents et volontaires, libres et disciplinés». Inversement, «l'être conscient et volontaire est celui qui fait et observe des conventions »; l'être conscient implique donc la convention (ibid., p. 18). De façon plus générale, on se trouve ici en présence d'un mode de représentation philosophique fondé sur «l'étude des rapports de correspondance ou de 
combinaison entre plusieurs termes» dans le cadre duquel les agents «ne sont définis et expliqués que par leurs rapports eux-mêmes». Le conventionnalisme ainsi proposé «est donc un ordinalisme», à savoir une philosophie qui concentre son attention sur «l'étude des combinaisons ou des correspondances de plusieurs groupes ou séries de termes, dont chacun ne prend un sens et une valeur que dans ces correspondances mêmes» (ibid., p. 23). Appliquée à l'étude de la vie en société, cet ordinalisme philosophique débouche sur une conception dialectique très actuelle de la relation entre l'individuel et l'institué. Celle-ci nous suggère l'idée de l'individu, être social, à la fois produit du social et producteur de social. Sur ce point, il y a convergence avec George Herbert Mead qui, à la même époque, s'intéressait à la constitution progressive de la personnalité dans la dynamique des relations interpersonnelles (Mead, 1934, 1962). Pour Dupréel comme pour Mead, l'individu social se construit au travers des contacts avec autrui, dans un dialogue intérieur entre la composante réflexive de sa personnalité et sa composante adaptative. Cette conception dialectique introduit également une sociologie pluraliste à caractère relationnel dont on redécouvre actuellement le potentiel théorique. Comme le notait récemment Pierpaolo Donati, on peut être d'avis que «l'objet de la sociologie n'est ni le 'sujet', ni le système social, ni aucun des couples similaires ... mais la relation sociale» (2004, p. 237). Cette sociologie relationnelle se situe à égale distance des conceptions holistes privilégiant des entités supra-individuelles et des conceptions individualistes réduisant les phénomènes collectifs à des effets d'agrégation.

\section{LE PLURALISME SOCIOLOGIQUE ET LE RAPPORT SOCIAL}

Pour Dupréel, la notion de société ne peut être conçue ni comme une somme d'éléments ni comme une synthèse d'éléments. Il serait vain de nier que la société est la somme de ses membres mais il serait tout aussi vain de refuser l'idée que la réalité sociale est faite «d'individus répartis dans un certain ordre», cet ordre subsistant au renouvellement de ses membres (1948, p. 3-4). L'ordre social ainsi conçu n'a rien de substantiel: il est constitué de rapports dont les individus sont les termes et qui s'agencent eux-mêmes en «rapports de rapports ». La sociologie peut être définie comme l'étude de ces relations et le rapport social en est la notion fondamentale. A cet égard, Dupréel se situe dans le prolongement de Guillaume De Greef (1842-1924) et d'Emile Waxweiler (1867-1916), autres figures marquantes de l'Ecole sociologique de Bruxelles. Comme chez De Greef ou chez Waxweiler, on note ici la recherche d'une catégorie élémentaire qui permette l'élaboration de propositions généralisantes, assortie de la mise en évidence d'un élément moteur de la dynamique sociale. L'unité d'analyse est toujours une catégorie d'interaction. L'élément moteur, c'est la force chez De Greef, l'énergie chez Waxweiler, la complémentarité dans l'interdépendance chez Dupréel (Coenen-Huther, 1997, p. 93-95). Le concept de rapport social n'est pas nouveau au moment où Dupréel en fait la base de son édifice théorique. Mais il constitue à ses yeux une généralisation de notions antérieures, présentes chez Tarde, chez Durkheim ou chez Giddings. Le premier voit dans l'imitation le fait social le plus caractéristique. Le second accorde une importance primordiale aux 
pressions que la collectivité exerce sur l'individu. Pour le troisième, le fait social élémentaire réside dans la conscience d'appartenir à une même espèce (consciousness of kind). Dans chaque cas, le social est défini par une espèce particulière de rapports sociaux. Tel que le conçoit Dupréel, le rapport social est une catégorie formelle, indépendante du type d'actions ou d'états d'esprit qui y sont impliqués. Rapports sociaux d'imitation, rapports sociaux définis par la pression de la collectivité ou rapports sociaux définis par le parallélisme des états d'esprit (like-mindedness) sont des catégories particulières de rapports sociaux (1912, p. 35-40). L'essentiel réside dans la notion même de rapport. Sur ce point, Dupréel se réfère à Simmel (ibid., p. 42, note 1) et insiste sur sa parenté de pensée avec Bouglé (ibid., p. 39) lorsque celui-ci écrit: «Peut-être (...) faut-il remonter jusqu'à l'idée même de rapport, et tenir pour un fait social tout ce qui résulte des rapports quels qu'ils soient qui s'établissent entre les individus ...» (Bouglé, 1896-1897, p. 152). Dans sa Sociologie générale (1948), Dupréel pose qu'il existe un rapport social entre deux individus «lorsque l'existence ou l'activité de l'un influe sur les actes ou sur les états psychologiques de l'autre. Une influence réciproque, actuelle ou virtuelle est le cas normal» (ibid., p. 5). Depuis Le rapport social (1912, p. 30), la formulation de cette définition a peu varié si ce n'est pour donner à la phrase un tour plus lapidaire et plus général. Dans la publication posthume de Similitude et dépassement, les «états psychologiques » sont remplacés par «la manière d'être» $(1968, \text { p. } 77)^{5}$. L'influence qui s'exerce dans le rapport social peut se manifester de trois façons: par la force ou la contrainte, par la persuasion au sens le plus large ou encore par l'échange d'avantages. Ces trois modes d'influence seront à la base d'une typologie des groupes sociaux. La capacité d'exercer une influence - que ce soit par la force, par la persuasion, par l'échange ou par une combinaison de ces procédés - est la force sociale qui est inégalement répandue. On peut opérer une distinction entre rapports sociaux positifs, impliquant un accord des deux termes, et rapports sociaux négatifs, comportant un certain antagonisme. Tout rapport social positif augmente la force sociale de chacun de ses termes; le rapport négatif, au contraire, est destructeur de force sociale.

\section{DU RAPPORT SOCIAL À LA STRUCTURE SOCIALE}

Les rapports sociaux s'interpénètrent et comportent des termes communs. Lorsque deux rapports sociaux ont un terme commun, on pourra dire que «l'un de ces rapports est complémentaire de l'autre s'il en conditionne soit l'existence, soit la nature» (Dupréel, 1948, p. 17). La complémentarité est le plus souvent réciproque. Deux rapports sociaux mutuellement complémentaires A-B et A-C déterminent un troisième rapport $\mathrm{B}-\mathrm{C}$. La complémentarité de deux rapports sociaux «entraîne logiquement une combinaison de trois rapports ». L'implication mutuelle des rapports complémentaires «se prolonge en des chaînes de longueur

\footnotetext{
Par «états psychologiques», Dupréel entend «les connaissances, les sentiments et les volontés » (1948, p. 5).
} 
indéfinie et leurs implications terme à terme se multiplient en réseaux inextricables» (ibid., p. 18-19). De manière générale, la complémentarité positive augmente la force sociale du terme moyen. Si l'influence d'un rapport complémentaire A-B renforce le caractère positif d'un rapport influencé A-C, ce rapport $\mathrm{A}-\mathrm{B}$ devient « un facteur d'agrégation du troisième terme $\mathrm{C}$ au terme moyen $\mathrm{A}$ » (ibid., p. 20). Ainsi, la complémentarité positive est à la base de la notion de groupe social, à savoir «une collection d'individus unis entre eux et distingués des autres individus par des rapports sociaux positifs et complémentaires » (ibid.). Tout comme les rapports sociaux, les groupes sont capables d'interpénétration, c'est-à-dire qu'ils peuvent avoir des termes communs. Seuls les groupes mutuellement extérieurs peuvent être définis par la différence des individus qui les composent. Lorsque les groupes s'interpénètrent, ils ne se différencient que par «la non-coïncidence de leurs structures, c'est-à-dire du système de rapports complémentaires communs à leurs membres respectifs» (ibid., p. 43). C'est par leur interpénétration que les groupements de toutes sortes en viennent à se trouver dans des rapports de complémentarité fondés sur une certaine mesure de spécialisation. En raison de la diversité des formes d'activité, les groupes sont beaucoup plus différenciés que ne peuvent l'être les individus. Les groupes ne sont pas des ensembles complets: ils ne subsistent qu'en état de complémentarité avec d'autres groupes. L'ensemble des groupements de toute nature, s'interpénétrant pour la plupart, est qualifié de symbiose sociale. Avec le rapport social et le groupe, la symbiose sociale est pour Dupréel une des trois notions fondamentales de la sociologie, dont les autres ne font que dériver. Une symbiose est distincte des groupements qu'elle inclut en ce sens qu'elle reste hétérogène du point de vue de la structure comme du point de vue de la force sociale. Elle comporte dès lors « un minimum de désordre et d'antagonisme» (ibid., p. 48).

Le projet scientifique d'une sociologie se construisant à partir de la notion de rapport social a fait l'objet de critiques et a donné lieu à des malentendus. Pour Gurvitch, Dupréel avait commencé, dans Le rapport social de 1912, par définir la sociologie «comme l'étude des différents rapports sociaux». Ensuite, dans sa Sociologie générale de 1948, il aurait «dépassé le formalisme» et admis que la sociologie peut se définir comme «la science des groupements sociaux» (Gurvitch, 1967, p. 6). George Davy, dans le compte rendu de la Sociologie générale qu'il donna à L'Année Sociologique, déclara qu'il «ne nous paraît pas possible de fonder, comme M. Dupréel semble trop enclin à le faire, la sociologie sur une science abstraite des rapports sociaux, science qui ramène fatalement à la psychologie individuelle» (Davy, 1948-1949, p. 130). Il est vrai que, pour Dupréel comme pour Simmel, «Il y a société là où il y a action réciproque de plusieurs individus» (Simmel, 1908, 1992, p. 43). Mais la sociologie ne s'est jamais limitée pour lui à l'étude des rapports sociaux, ce que ni Davy ni Gurvitch ne semblent avoir compris. Le rapport social est pour Dupréel la catégorie élémentaire sur laquelle se construit un édifice théorique et conceptuel qui part de la relation interindividuelle pour aboutir aux phénomènes macrosociaux. Dupréel est très conscient de ce qu'on appelle aujourd'hui les «effets émergents » de l'agrégation des relations élémentaires. Et le rapport social étant une catégorie relationnelle, il n'y a aucune raison de penser qu'il ramène à la psychologie individuelle, même si les analyses sociologiques s'établissent toujours selon Dupréel 
«sur un fond de psychologie, perceptions, émotions, instincts, affections, fins conçues, passions, moyens reconnus, raisonnements, informations, connaissances, résolutions » $(1948$, p. 7). Gurvitch s'étonne de ne trouver dans la Sociologie générale «aucune conscience de l'irréductibilité des groupes et de la société en tant que réalités indécomposables » $(1967$, p. 6). Mais si l'ordre social est pour Dupréel «une réalité irréductible au contenu d'une conscience ou d'une pluralité de consciences » $(1948$, p. 4), sa sociologie évite précisément tout holisme et ne considère nullement les groupements sociaux de tous ordres comme des « réalités indécomposables ».

Un groupement qui se développe dans la durée, nécessairement, se structure. L'existence d'une structure est la conséquence de la complémentarité. La structure d'une société quelle qu'elle soit est «l'ensemble des rapports sociaux complémentaires durables ou permanents » qui oriente les individus les uns vers les autres (ibid., p. 100). Dans tout groupement plus ou moins organisé, la vie sociale fait «de tout rapport social qui s'institue un rapport à trois termes au moins, les deux parties plus un tiers, allié de l'une des parties, ou garant de chacune d'elles ». La structuration provoquée par la complémentarité entraîne une véritable «organisation de la complémentarité». Des individus en position de troisième terme tendent à devenir des «spécialistes» des rapports complémentaires. Ce sont des notables ou ils le deviennent: leur force sociale dépassera «la moyenne de capacité d'influence dévolue aux simples particuliers». Ainsi se constitue la structure fondamentale de tout groupe. Il y a d'une part «la masse des particuliers», d'autre part les individus auxquels on reconnaît «la prérogative d'intervenir en tiers dans les rapports positifs et négatifs des particuliers». Ces derniers seront en position de supériorité du fait de leur «surcroît de capacité d'influence». Dupréel en tire une proposition à caractère tout à fait général: la complémentarité porte en germe une asymétrie dans les rapports. L'institution de chefs ou de dirigeants, tout comme celle de juges, peuvent être considérées comme des cas particuliers «du jeu de la complémentarité». Les fonctions de «spécialistes de la complémentarité» tendent à se concentrer sur les mêmes personnes. Il se crée ainsi un «noyau structural» formé d'individus bénéficiant d'une certaine prééminence «grâce aux rôles de direction, de contrôle, d'arbitrage, de garantie qu'ils assument». Ce noyau tend à se comporter comme un groupe particulier vis-à-vis du reste de la société. Le jeu de la complémentarité, tel qu'il est analysé par Dupréel, est ainsi à la base d'un mécanisme de constitution d'élites se distinguant de la masse. L'influence du noyau structural sur la masse prend l'une des trois formes déjà mentionnées : «contrainte par la force, entraînement par la croyance et les prestiges, ascendant par libéralités ou échanges consentis ». C'est à ces phénomènes de structure que sont liées la formation des classes sociales et les modifications de leur consistance (ibid., p. 101-103). Au plan de la société globale, ces phénomènes de structuration donnent lieu à la mise en place d'une «structure en quadrillé». Celle-ci peut être schématisée sous forme « de figures oblongues, verticales et parallèles » représentant des domaines d'activité tandis que «d'autres figures allongées, sortes de bandes ou de rubans traversant horizontalement les premières », représentent des classes ou des niveaux de statuts (ibid., p. 125). L'interdépendance des relations s'organise de façon de plus en plus intersectorielle à mesure qu'on s'élève dans les différentes colonnes 
représentant les différents secteurs de la hiérarchie sociale et que s'élargit le champ des tâches de coordination. En d'autres termes, la complémentarité des rapports sociaux déborde de plus en plus les domaines d'activités spécifiques à mesure qu'on s'élève dans leurs hiérarchies respectives. Ainsi se trouve amorcée la tendance à l'oligarchisation 6 .

On a voulu voir dans Le rapport social de 1912 un «manifeste antidurkheimien» (Croissant, 1968, p. 208). Ceci est une erreur d'appréciation. Certes, Dupréel a clairement cherché à définir une orientation théorique originale par rapport au psychologisme de Tarde et au sociologisme attribué à Durkheim. Il voit en particulier dans la notion de rapport social une manière « de se débarrasser de l'encombrante préoccupation de la société prise comme un tout» (1912, p. 40). Il n'en indique pas moins tout aussi clairement ce qui le rapproche de Durkheim. «M. Durkheim», écrit-il, «insiste, non sans raison, sur la nécessité de traiter les faits sociologiques comme des choses, c'est-à-dire autrement que dans les représentations qu'une conscience peut s'en faire (...) Rien ne répond mieux à ce desideratum que le rapport social» (ibid., p. 38). Mais il est plus important de bien comprendre la nature du projet sociologique de Dupréel, fondé sur la notion de rapport social. Ce projet s'inspire philosophiquement de Kant et sociologiquement de Simmel. Il s'agit ni plus ni moins que d'explorer les conditions a priori de la vie en société. Le rapport social est une catégorie formelle. Cette forme devient une réalité empirique lorsqu'on la trouve «ordonnant les démarches des êtres vivants »; il suffit pour cela que ceux-ci soient mutuellement influençables. Cette forme, écrit Dupréel, «impose à cette réalité ses propriétés». Il est véritablement question «d'une sociologie formelle a priori». Il y a des lois de l'association qui s'imposent quelle que soit la nature des associés: «compatibilité mutuelle, concessions réciproques, combinaisons d'avantages». Il y a d'autre part des «conditions d'existence des antagonismes, de leur durée, de leur évolution, aggravation, atténuation, etc.» (1948, p. 9). Ces règles formelles peuvent être dégagées par l'observation de la réalité sociale mais les plus générales peuvent être posées d'avance «et comme par déduction». Et ceci amène Dupréel à la déclaration provocante contre laquelle s'est élevé le Doyen Davy (1948, p. 130; 1968, p. 133): «on peut faire la Sociologie de Sirius sans tomber dans la fantaisie» $(1948$, p. 9).

\section{LE RAPPORT SOCIAL ET LES INSTINCTS SOCIAUX}

La référence de Dupréel à une éventuelle « sociologie de Sirius » est loin d'être une boutade. Elle contient l'affirmation que les conditions formelles de la vie en société doivent pouvoir s'appliquer quelle que soit l'espèce des individus et leurs particularités. Il y a rapport social - donc amorce d'une vie sociale - dès que deux êtres vivants sont en mesure de s'influencer l'un l'autre. La sociologie humaine

' Sur la «structure en quadrillé » comme support de tendances à l'oligarchisation, voir: CoenenHuther, 2004 (p. 37-44). 
émerge donc d'une sociologie des espèces animales. Comme on va le voir, cette constatation n'implique, chez Dupréel, aucun réductionnisme biologique ou psychologique; elle prend néanmoins pour base le fait que l'espèce humaine est aussi une espèce animale et qu'il y a des conclusions à en tirer. A moins de postuler «un créationnisme du type biblique» sans aucun fondement scientifique, il paraît raisonnable de considérer que l'être humain est issu «d'ancêtres déjà sociaux avant d'être humains» (1948, p. 51; souligné par JCH). En d'autres termes, l'individu social est préalable au fait de l'association humaine. Il serait biologiquement et sociologiquement absurde de considérer la vie en société comme «un phénomène tardif, préparé par une évolution des individus isolés ». Il existe dès lors des instincts sociaux assurant «une aptitude et une propension à la vie en société » transmises par voie héréditaire, de la même façon qu'il y a un instinct de conservation qui favorise la durée de la vie individuelle. Bien que l'être humain soit un être pauvre en instincts, il est sujet à des réactions instinctives comme les autres espèces animales. A la différence de celles-ci, l'espèce humaine est cependant « douée de mémoire, de volonté dirigée, de la capacité d'apprendre et d'inventer». Il se crée ainsi une situation conforme à la spécificité de l'espèce humaine. L'aptitude humaine à la pensée réflexive l'emporte largement sur une organisation instinctuelle qui reste réduite. Nos conduites instinctives se conjuguent avec une activité raisonnée ou s'opposent à elle. L'activité instinctive et l'activité raisonnée sont sous l'influence des «conditions générales de la vie en association» (ibid., p. 49-51). Dupréel en conclut à la nécessité de distinguer une «paléosociologie» et une «néosociologie».

La paléosociologie traite de «tout ce qui, dans l'activité des êtres sociaux, dépend directement des tendances organiques, héréditaires, appropriées ou favorables à la vie en société». Il s'agit de l'étude des instincts sociaux et de leur rôle dans les associations d'individus (ibid., p. 50). La néosociologie traite exclusivement de l'espèce humaine. Elle a pour objet l'activité sociale dans laquelle «vient se combiner avec l'apport des instincts tout ce qui résulte du rôle des facultés supérieures, éducabilité, langage, mémoire, imagination, invention, raison ». Ceci l'amène à prendre en compte «les structures des rapports sociaux complémentaires, la multiplicité, la diversité et l'interpénétration des groupes, le progrès indéfini des techniques et des consciences (ibid.) Pour Dupréel, l'étude des sociétés humaines se doit de combiner les acquis de la paléosociologie et de la néosociologie. Les instincts sociaux ne doivent pas être relégués dans l'étude des espèces animales. Ils sont au nombre des données de la sociologie humaine car «nul progrès des facultés supérieures n'abolira jamais le rôle des instincts et des tendances organiques » (ibid.) Il importe cependant - pour éviter des malentendus regrettables - de préciser à ce propos que Dupréel rejette absolument tout primat de l'organique et de l'hérédité. L'idée d'une telle primauté repose selon lui sur une confusion entre problèmes de nature et problèmes d'origine. L'origine de toute vie sociale ne peut être recherchée que dans les lents progrès initiaux des tendances organiques. En revanche, la nature de la vie sociale ne peut être élucidée qu'à partir de la notion de rapport social. Toute vie en société doit être conforme aux propriétés des rapports sociaux car il y a des règles de compatibilité et d'incompatibilité qui s'imposent à tout groupement, qu'il soit animal ou humain. Les conditions formelles de la vie en société constituent « la logique interne du social» 
et s'imposent aux individus qui vivent ensemble, quelle que soit leur évolution organique ou psychologique (ibid., p. 50-51).

Il n'y a pour Dupréel aucune contradiction entre l'idée de sociologie formelle et celle de l'existence d'instincts sociaux. Les instincts sociaux orientent vers la vie en groupe et font de l'espèce humaine une espèce grégaire. En ce sens, l'être humain fut naturellement social avant de l'être culturellement. Mais la vie en groupe et, de façon plus générale, la vie en société, est soumise à des conditions formelles. Celles-ci interviennent à un niveau général et abstrait. Elles se concrétisent et se chargent d'un contenu spécifique dans les différentes sociétés ou cultures empiriquement observables. C'est alors qu'interviennent la production de social et la convention, ce qui aboutit à des comportements diversifiés. Naturalisme social et constructivisme social se combinent donc chez Dupréel dans une perspective évolutionniste.

La théorie des instincts sociaux qui semblait devenue désuète rencontre actuellement un regain d'intérêt à la faveur du progrès rapide des connaissances en éthologie. On a longtemps vécu sur l'idée d'une frontière bien nette entre le genre humain et le reste du règne animal (Lestel, 2001, p. 15). A l'heure actuelle, on tend de plus en plus à en revenir à la conception de Dupréel selon laquelle l'être humain, bien que caractérisé par un potentiel d'autonomisation culturelle vis-àvis de la nature, est le produit de l'évolution naturelle des espèces (ibid., p. 162). Cette vision de l'humain comme produit d'un processus évolutif suggère que des stades intermédiaires peuvent exister entre les conduites purement instinctuelles et les comportements marqués par la réflexivité. Cela nous suggère également que des traces d'animalité subsistent dans les attitudes et les comportements des êtres humains d'aujourd'hui. Pour certains auteurs, des constantes plongeant leurs racines dans une animalité pré-humaine seraient à la base d'un sens moral ancré dans la nature plutôt que dans la culture ${ }^{7}$. Rien dans l'œuvre de Dupréel ne peut être invoqué à l'appui de cette conception. Pour lui en effet, l'acte moral est un acte réfléchi, volontaire, impliquant un sacrifice consenti ou, à tout le moins, un détriment volontairement accepté ${ }^{8}$. Penser un acte comme acte moral - à commencer par la convention - exige l'existence d'un individu qui en ait l'initiative consciente et qui ne se contente pas d'obéir à un instinct ou à un réflexe. Que cette capacité existe à l'état d'ébauche et de promesse chez d'autres espèces vivantes ne lui enlève aucunement sa caractéristique de condition impérative. L'acte moral, pour Dupréel, suppose liberté, responsabilité et capacité de débat avec soi-même. Ces ingrédients sont absents des comportements «sociaux» ou «altruistes » observables chez certaines espèces animales ${ }^{9}$.

Voir à ce sujet l'ouvrage de J. Q. Wilson, The Moral Sense (1993) et le commentaire qu'en donne Raymond Boudon dans Le juste et le vrai (1995).

s C'est également la position de Ferry et Vincent (2000, p. 49 et sq.).

- J'ai déjà eu l'occasion d'aborder ces questions dans des publications récentes (Coenen-Huther, 2001 et 2003). 


\section{DE L'ORDRE SOCIAL À L'ORDRE DES VALEURS}

Un problème que Dupréel tient pour commun à la sociologie et à la philosophie est celui des rapports entre la hiérarchie sociale et la hiérarchie des valeurs (1948, p. 310). La défense ou la contestation d'une hiérarchie sociale fait appel à des jugements de valeurs. La justification de l'ordre établi se fonde sur l'idée de coïncidence entre la supériorité sociale et l'excellence ou la supériorité dans la conformité à l'une des valeurs célébrées universellement: le vrai, le beau, le bien. De même, la protestation contre les injustices de l'ordre établi cherche à montrer que la hiérarchie sociale «viole l'ordre des bonnes valeurs » (ibid.) La revendication qui s'exprime contre une hiérarchie établie peut s'appuyer sur des valeurs communes. Les valeurs proclamées par la classe supérieure ne sont pas remises en question mais on s'efforce d'en montrer le caractère illusoire pour les couches défavorisées de la population. La contestation peut également tirer argument d'un « désaccord sur les valeurs ». Dans ce cas, « la lutte pour le classement se fait ... sur le plan de la hiérarchie des valeurs en elles-mêmes »; il y a tentative de renversement des valeurs consacrées (ibid., p. 311). Pour l'observateur dépourvu de préjugés, il y a d'incontestables rapports entre l'ordre social et l'ordre des valeurs mais aucune hiérarchie sociale ne sera jamais entièrement et définitivement conforme à une échelle de valeurs communément acceptées. Aucune répartition de la force sociale ne peut passer «pour entièrement juste» (ibid., p. 314) et la coïncidence de la prééminence et de l'excellence sera toujours contestable. Il en résulte qu'une échelle de valeurs peut servir autant à protester contre les inévitables injustices de l'ordre établi qu'à maintenir ou à instaurer un ordre social (ibid., p. 315).

Pour Dupréel, l'ordre des valeurs, comme l'ordre social, participe de la consistance du réel et de la précarité du conventionnel. La valeur se caractérise par un dualisme foncier: elle doit sa consistance à ce qu'elle relève d'un ordre; sa précarité résulte de l'intervention contingente d'une force (1939, p. 119). La valeur morale, pour prendre cet exemple, est caractérisée tant par la consistance que par la précarité. Le mérite moral résulte du respect d'une règle, règle qu'on accepte ou règle qu'on se donne. La conduite morale ne varie donc pas comme peuvent varier les intérêts ou les passions: elle présente une consistance propre. Il arrive que «la conscience accepte la persécution pour demeurer fidèle à un devoir qu'elle juge inconditionnel.» A l'opposé, la conduite «déréglée » est «inconsistante » car «elle est tout entière soumise aux variations du désir ou du besoin» (1939, p. 84-85). Une valeur morale élevée est liée à « un degré de consistance » que ne présente pas «la conduite strictement honnête» (ibid., p. 85). Mais la valeur se caractérise également par sa précarité. L'acte moral résulte d'une «combinaison de l'ordre, de la règle et de la force du sujet» mais cette combinaison n'est en rien «nécessaire » et l'action morale n'est jamais que «probable»; elle a un caractère précaire. La valeur morale ne se discerne que lorsque «la liberté d'agir» empêche de la considérer comme assurée. De façon plus générale, «la valeur est précaire par le fait qu'il n'y a de valeur que par un sujet» (ibid., p. 90-91).

Tout comme l'appartenance à un groupe ne s'impose à la conscience que par contraste avec d'autres groupes, une valeur ne peut être discernée que «par 
opposition à d'autres valeurs » auxquelles elle est préférée. Les valeurs sont donc multiples, tout comme le sont les allégeances de groupes, et les tentatives de les ramener à une valeur unique ne peuvent conduire qu'à des absurdités. L'idée de l'unicité de la valeur est en effet contradictoire dans les termes car elle ferait disparaître les deux caractéristiques fondamentales de la valeur: la consistance et la précarité. On retrouve ici le principe général selon lequel l'idée d'un ordre unique équivaut à la dissolution de la notion d'ordre. Poser la valeur comme «quelque chose d'unique et d'identique à soi-même» exigerait qu'on fasse appel à une «idée-béquille ou complémentaire» qui serait «l'idée de non-valeur ou fausse valeur». Cependant, ces «fausses valeurs» ne peuvent qu'être conceptualisées comme des valeurs «puisqu'elles sont capables de déterminer nos actes et nos sentiments». Entre valeur vraie et «fausse valeur», c'est la valeur préférée qui devient aux yeux de l'individu la «vraie valeur». Mais en considérant cette valeur comme «quelque chose d'unique et d'identique sous toutes ses manifestations» on en fait quelque chose d'indéterminé. Or, une réalité indéterminée ne peut donner lieu à une idée cohérente; elle est aussi inconsistante que «la matière sans forme ou l'énergie sans direction». En outre, la considérer comme unique revient à lui conférer une existence nécessaire: il ne pourrait en résulter qu'une absence de précarité (ibid., p. 99-103). La multiplicité des valeurs est donc irréductible. Les valeurs sont également «relatives» car aucune d'entre elles «ne saurait être posée en dehors de son rapport avec d'autres valeurs» (ibid., p. 99-101).

La vie sociale est faite d'opérations de «transport» de valeurs et de «promotion» de valeurs. Le transport de valeur résulte de la découverte que «la valeur considérée dans un certain objet est la même que l'on tient pour établie dans un autre objet» (ibid., p. 105). La promotion de valeur consiste dans la proclamation de la valeur d'un objet (ibid., p. 107). La distinction classique entre jugements de valeur et jugements de réalité peut être exprimée en ces termes. Le jugement dit de valeur est avant tout une opération de promotion de valeur; le jugement dit de réalité est principalement une opération de transport de valeur. La différence entre ces deux types de jugements ne réside cependant ni dans la forme des propositions formulées ni dans la nature des termes utilisés; elle résulte du rôle que celui qui formule des jugements leur fait jouer dans son $\operatorname{propos}^{10}$ (ibid., p. 112-113). Plus généralement, les conditions d'existence des valeurs sont inséparables des rapports entre individus et entre groupes. Elles sont en effet équivalentes «à leurs conditions d'affirmation». En d'autres termes, «dès qu'une valeur est affirmée, elle est $»$ : si elle n'avait pas une certaine consistance permettant de la discerner, elle ne serait pas affirmée; si elle n'était absolument pas précaire, elle serait considérée comme allant de soi et ne ferait l'objet de manifestations d'allégeance d'aucune sorte (ibid., p. 120). Il en résulte que les valeurs ne se démontrent pas: elles se posent et elles s'imposent. Elles s'imposent, précisément parce qu'elles sont des valeurs (Coenen-Huther, 2001, p. 254). Les valeurs se hiérarchisent en quelque sorte par définition. Affirmer une valeur, c'est l'opposer implicitement ou

${ }^{10}$ Comme le fait observer Dupréel, la phrase «Du Bartas est un poète» peut être un jugement de valeur ou un jugement de réalité selon l'intention du locuteur (Dupréel, 1939, p. 113). 
explicitement à une autre valeur et la tenir pour supérieure. Au sommet de la hiérarchie des valeurs, on trouvera des valeurs qu'on peut qualifier d'absolues: le Bien, le Beau, le Vrai ${ }^{11}$. Ces valeurs ont une «consistance portée au maximum »; leur consistance «n'est soumise aux vicissitudes d'aucune autre valeur». Elles n'en restent pas moins précaires: «d'autres valeurs peuvent toujours leur être préférées » et ce sont elles qui sont le plus directement en butte aux tentatives illusoires de restaurer l'unicité de la valeur (Dupréel, 1939, p. 199-207). La reconnaissance des valeurs absolues n'est fondée «ni sur un ordre nécessaire, ni sur une force irrésistible». Elle est fondée sur «une convergence des activités relativement probable»; cette synthèse d'ordre et d'activité «est toujours éminemment précaire » (ibid., p. 212). Il s'agit de valeurs «qu'aucune variable ne fait fléchir ou ne conditionne» et qui commandent des attitudes très tranchées: «obéir à la règle quoi qu'il arrive, reconnaître la beauté en dehors de toute utilité, apercevoir la vérité comme supérieure à toutes les convenances et à tous les intérêts ». La précarité est néanmoins toujours menaçante «puisque son attachement à des choses, à des êtres, à des groupes peut toujours détourner une conscience de telle valeur inconditionnelle et l'inciter à défavoriser une vérité, dégrader une beauté, faire violence à un idéal» (ibid., p. 204-205).

Dans l'optique de Dupréel, les valeurs absolues, à la charnière de l'ordre et de l'action, se dégagent de trois phases différentes de développement de la conscience humaine qui sont envisagées dans une perspective à long terme et qui suggèrent une autonomisation graduelle de l'ordre des valeurs par rapport à l'ordre social. Le Bien, comme valeur morale absolue, émerge tout d'abord de la dynamique des instincts sociaux. Au degré suivant, les comportements de nature biologique sont modifiés en fonction «de la convenance du groupe social». En ce sens, le social discipline le biologique ou s'oppose même à lui. La valeur de l'acte respectueux de la norme reste relative puisqu'il s'agit de l'observance d'une règle «fondée sur l'intérêt de la société». Enfin, dans la phase «de l'idéal moral», on a le sentiment que la règle doit être respectée «parce qu'elle est bonne en ellemême». Elle devient un impératif catégorique, indépendant de toute considération de conformité ou d'utilité sociale. Ainsi s'amorce la dissociation de l'idée de valeur et de «l'idée de convenance ou d'avantage». Il en résulte l'idée d'une valeur pure ou valeur absolue, «posée en dehors de toute opération de transport qui lierait sa qualité de valeur à quelque autre valeur préalablement reconnue » (ibid., p. 210).

L'examen de la valeur du Beau conduit à une analyse semblable, en termes de gradation des valeurs esthétiques. Par analogie avec la conscience morale, Dupréel parle d'une «conscience esthétique » qui traverse des phases de développement successives. Son premier degré, relevant également des instincts sociaux, correspond à la recherche de sensations agréables: un plaisir esthétique pur. Le deuxième niveau pourrait être appelé celui de «la discipline du goût». La connaissance y joue «un rôle de contrôle sur l'impulsion brute». On se réfère alors à des

${ }^{11}$ Comme toutes les valeurs, ces valeurs sont relatives lato sensu. Elles peuvent néanmoins être qualifiées d'absolues, par opposition à des valeurs relatives stricto sensu (Dupréel, 1939, p. 99). 
normes reconnues. L'objet apprécié est «l'objet correct, à la mode, travaillé selon le style établi». Ces normes sont «des règles de groupes» et l'adhésion comporte un élément de distinction sociale. Le troisième niveau de la conscience esthétique est celui « de l'art et de la beauté pure». La valeur de symbole de l'œuvre d'art «se perd à mesure que le style, étant plus universellement adopté, cesse d'être le fait du groupe qui en a assuré d'abord le succès ». Cet état supérieur de la conscience esthétique constitue une synthèse des deux premières phases. Il s'y ajoute toutefois un élément de connaissance supplémentaire: on sait que l'objet admiré a une valeur en soi, ce qui confère une «assurance spécifique» à la conscience esthétique. La consistance de l'objet reconnu beau est «au maximum» car sa valeur n'est plus fonction d'aucun autre critère d'appréciation, tel que l'utilité, l'avis de son propriétaire, le prix qu'on peut en obtenir ou quelque mode passagère (ibid., p. 212-217).

Jusqu'à un certain point, l'émergence de la valeur du Vrai peut être analysée de la même façon, à l'aboutissement des progrès de la faculté de connaître. A l'origine, il y a le perfectionnement d'une activité toute animale, enchaînant des moyens en vue d'une fin. La connaissance n'arrive toutefois «à la plénitude de sa nature» que lorsqu'elle peut être «détachée de l'objet et de l'occasion de ses applications ». Cette deuxième phase du développement de la faculté de connaître exige qu'elle soit «exprimable et intelligible». Elle suppose l'existence d'un langage et implique un groupement social. Elle requiert également l'aptitude à établir des conventions, en l'occurrence des accords sur les connaissances acquises (ibid., p. 218-219). Mais la connaissance vraie doit être distinguée de toute connaissance simplement communicable. Car «perception et croyances communes sont des valeurs relatives, liées à leurs effets» tandis que la vérité est une valeur absolue: «une proposition réputée vraie a une consistance propre: la raison de l'affirmer n'est plus du tout sous la dépendance d'un but pratique à atteindre, d'une convenance à respecter ». Dans la troisième phase de développement de ce que Dupréel appelle la «conscience théorique», l'idée de connaissance se dégage de toute considération d'ordre utilitaire. Une connaissance est vraie «malgré qu'elle soit inutile, nuisible, contestée ou méconnue» (ibid., p. 219). C'est «une connaissance confirmée»; elle dépend uniquement de «la possibilité de la vérifier».

De la rupture entre le vrai et l'utile résulte l'unicité de la vérité (ibid., p. 220-222). De ce point de vue, la valeur de Vérité s'écarte de celles du Bien et du Beau. Dupréel fait observer que ces dernières comportent des antinomies internes. Les conflits de devoir en constituent l'illustration la plus claire pour ce qui est du Bien. L'esthéticien est également confronté à des dilemmes qui relèvent de l'antinomie interne à la valeur du Beau. Face à un tableau ancien, qu'est-ce qui importe: l'intention du peintre - qui impose un lavage - ou la patine du temps qui s'est incorporée à l'œuvre? (ibid., p. 237-239). La valeur du Vrai, au contraire, ne peut comporter aucune antinomie car elle n'existe que par son unicité. Le beau et le bien, tout comme la connaissance en général - à distinguer de la connaissance vraie - sont des valeurs d'action ou de réalisation; elles ont donc une précarité qui leur est propre en ce sens qu'elles dépendent de contingences liées à l'action. La vérité est «entièrement détachable de l'action». C'est ce que Dupréel qualifie de «valeur d'intention ou d'aspiration» (ibid., p. 240-245.) 
Certains auteurs ont cru voir une contradiction entre le pluralisme ordinal de Dupréel et ce qui leur apparaît chez lui comme une hiérarchisation implicite des valeurs, favorisant le devoir moral face aux intérêts et aux passions. Pour Goriely, il est vrai que «l'ordre de la conduite morale a une consistance spécifique par rapport à celui des instincts ou des intérêts » mais il ne s'ensuit pas que cette consistance soit plus élevée. L'inverse, ajoute Goriely, «supposerait l'unicité d'un ordre hiérarchique, d'un ordre des ordres » (1968, p. 98). Genard reprend à son compte la remarque de Goriely et estime que Dupréel souscrit implicitement à une «hiérarchie transcendante des valeurs» d'inspiration kantienne (1995, p. 45). En fait, la contradiction apparente se résout par l'ancrage sociologique de la philosophie des valeurs de Dupréel. La conduite morale est porteuse d'universalité et les arguments en sa faveur sont recevables par un «auditoire universel ${ }^{12}$. Elle a une stabilité que ne peut atteindre la conduite inspirée par des désirs ou des intérêts. Son surplus de consistance lui vient donc du rôle qu'elle joue au profit de l'ordre social et de «l'accord des esprits». Un relativisme absolu impliquerait que l'intérêt ou le plaisir fournisse un principe hiérarchique équivalent à celui du devoir moral. Mais le relativisme de Dupréel n'est pas un relativisme absolu; c'est un relativisme tempéré par le souci de l'ordre social et du bien commun.

\section{DUPRÉEL, PHILOSOPHE ET SOCIOLOGUE DE L'ACTION}

Pour Dupréel, les valeurs absolues - le Bien, le Beau, le Vrai - se dégagent de l'activité humaine. Ceci ne pose aucun problème d'interprétation en ce qui concerne la valeur morale et la valeur esthétique. En revanche, si l'idée de vérité émerge également de l'action, elle acquiert une indépendance par rapport à son origine et c'est alors, comme on vient de le noter, le critère d'unicité qui la constitue en valeur. L'orientation de Dupréel vers l'action et vers les exigences de la vie sociale en fait un philosophe et sociologue de l'action. A ce titre, on peut être tenté de le ranger parmi les pragmatistes. Le pragmatisme constitue en effet une alternative à la pensée cartésienne centrée sur l'individu isolé, en dialogue mental avec lui-même. Il insiste au contraire sur le caractère intersubjectif de toute élaboration de connaissances et véhicule une conception relativiste de la vérité, mettant l'accent sur sa valeur pour l'action ${ }^{13}$. Il se fait que Dupréel connaissait bien le mouvement pragmatiste. Son maître, René Berthelot, le caractérisait comme un «romantisme utilitaire», à savoir «une théorie de la connaissance où l'opposition entre la vérité et l'erreur se définit par l'opposition entre ce qui est utile et ce qui est nuisible à la vie » (Berthelot, 1913, p. 3). Il y a incontestablement entre les conceptions de Dupréel et celles des pragmatistes une certaine affinité. Dans son Traité de morale, Dupréel qualifie le pragmatisme de «brillant mouvement philosophique» (1932, p. 269). Néanmoins, comme un commentateur l'a (1970).

${ }^{12}$ La notion d'auditoire universel est empruntée à Chaïm Perelman qui s'inspire de Dupréel

13 On pourra consulter à ce sujet l'ouvrage de Hans Joas, Kreativität des Handelns (1992), traduit en français sous le titre La créativité de l’agir (1999). 
fait observer, sa théorie de l'unicité de la vérité «met entre les pragmatistes et lui un abîme béant» (Barzin, 1968, p. 37). Au surplus, Dupréel ne peut accepter la réduction de l'idée de vérité à celle d'utilité (1939, p. 219). La divergence de vue réside selon lui dans une confusion entre «connaissance» et «connaissance vraie ». La connaissance en général, «vraie ou fausse, claire ou confuse », est pour lui une valeur relative qui peut prendre un caractère instrumental. La connaissance vraie est une valeur absolue qui se détache de toute considération utilitaire. Dupréel est à ce sujet très clair: «... nous ne saurions admettre que les vues exposées ici tendent à restaurer un pragmatisme. Le pragmatisme a été, et c'est son mérite, une philosophie des valeurs, mettant en pleine lumière l'importance de l'action; mais elle a échoué comme une mauvaise philosophie des valeurs ...» (1939b, 1949, p. 333-334, note 1, souligné par JCH). Un article antérieur, Les deux racines de la valeur du vrai (1935, 1949), fournit tous les éléments pour comprendre ce qui rapproche la pensée de Dupréel des conceptions pragmatistes mais aussi ce qui l'en sépare. L'argumentation développée débute par une interrogation: pourquoi accorde-t-on une «valeur éminente» à la connaissance vraie? Pour le sens commun, la vérité est précieuse parce qu'elle est utile. L'utilité est donc la première racine de la valeur du vrai. Mais la connaissance fausse peut également s'avérer féconde dans ses conséquences ou ses applications. Si donc l'utilité est une des racines de la connaissance vraie, ce n'en est pas une caractéristique spécifique; ce n'est qu'une «propriété possible» de toute connaissance, vraie ou fausse (ibid. p. 285-288). L'origine d'une connaissance peut être expliquée à partir de ses aspects utilitaires mais «la probabilité joue en faveur de la connaissance vraie», ce qui signifie que «la connaissance vraie est plus généralement ou plus probablement utile que la connaissance quelconque ». A ne s'en tenir qu'à ce critère, on pourrait penser que la différence entre vérité et «connaissance non dégrossie» est toute relative. Pourtant, «si, à certains égards, la vérité vaut mieux, à un autre point de vue la vérité vaut seule». Par rapport à la connaissance utile en général, la vérité est «d'un autre ordre» (ibid., p. 289-290, souligné par $\mathrm{JCH})$. Qu'un jugement soit considéré comme fécond n'implique pas qu'il soit unique. Au contraire, affirmer qu'un jugement est vrai, c'est affirmer que ce jugement «est seul à posséder une certaine valeur qu'on lui confère ». La vérité de ce jugement est une caractéristique dont tous les jugements concurrents sont «entièrement dépourvus». Et Dupréel de conclure: «croire à la vérité, c'est croire à cette unicité-là». La deuxième racine de la valeur du vrai réside donc dans cette «unicité spécifique», seule capable d'assurer l'assentiment général pour autant qu'il y ait eu convention sur les procédés de vérification. La connaissance en général est «une combinaison de connaissance et d'intérêt». En revanche, la connaissance vraie ne saurait dépendre ni d'intérêts ni de convenances: elle est connaissance pure et répond à un «idéal de vérité » (ibid., p. 291-293). Le vrai, en tant que connaissance, peut donc être «l'agent directeur de notre action» mais il est aussi « un facteur de l'accord des esprits». Bien que ces deux sources de valeur soient irréductibles l'une à l'autre, elles se combinent étroitement dans la vie et dans l'action. C'est de cette combinaison que résulte la valeur de la science. Celleci repose en effet sur une synthèse d'activités répondant à des demandes d'ordre pratique et d'autres répondant à un désir de connaissance pure (ibid., p. 296-297). Pour Dupréel, il y a donc bien ancrage de la valeur du vrai dans la vie sociale, mais ce qui sépare sa pensée des conceptions pragmatistes, c'est l'irréductibilité 
mutuelle des deux racines de la vérité. Pour William James, la vérité n'est rien d'autre qu'une croyance «qui donne satisfaction à l'ensemble de nos tendances individuelles, y compris les tendances intellectuelles ...» (1896). Pour Dupréel, au contraire, la recherche de la vérité implique «l'idée de renoncement ou de sacrifice » $(1935,1949$, p. 296). En ce sens, dans la mesure où elle se libère de son origine utilitaire, elle acquiert la dimension d'une quête morale.

\section{CONCLUSION}

Le rejet de l'idée classique de connaissance nécessaire est pour Dupréel une façon de s'opposer à une forme inadéquate d'affirmation de la valeur absolue de la connaissance vraie. En déconstruisant la notion philosophique de nécessité et en la décomposant en un principe statique et un principe dynamique, il évite les apories du déterminisme absolu et de la contingence pure et leur substitue un mode de pensée probabiliste qui conduit logiquement au pluralisme ordinal. La pluralité des ordres se traduit philosophiquement en une pluralité des valeurs et des échelles de valeurs. Elle se traduit sociologiquement en une pluralité des groupements et des allégeances dont la coexistence est assurée par la convention, acte créateur de social et acte spirituel, marquant le triomphe de l'esprit humain. L'ordre social n'a aucun caractère substantiel. Il est constitué d'un enchevêtrement des rapports qui s'établissent entre les individus. Et c'est de cet enchevêtrement de rapports qu'émergent les structures sociales par le jeu de la complémentarité. Il y a une logique du social qui est faite de conditions formelles se superposant aux tendances instinctives pré-humaines. La concrétisation de cette logique en une société ou une culture particulière comporte néanmoins une part de contingence qui la fait échapper au déterminisme. L'ordre des valeurs comporte un élément de précarité qui lui est propre. Il n'est en effet de valeurs que celles qui sont affirmées comme telles et dont certaines actions attestent la réalité. Mais la traduction d'une valeur en acte n'est jamais que probable. Des valeurs - le bien, le beau, le vrai - sont dites absolues car elles ne dépendent d'aucune autre valeur et ne peuvent se hiérarchiser entre elles. Elles n'en sont pas moins relatives lato sensu car on peut les opposer l'une l'autre et d'autres valeurs peuvent être proclamées pour les disqualifier. Le polythéisme des valeurs, assorti d'un sens moral fondé sur le renoncement, couronne l'édifice intellectuel qui se donne à voir dans l'œuvre philosophique et sociologique de Dupréel.

Département de sociologie

Université de Genève

Jacques.Coenen-Huther@socio.unige.ch

\section{RÉFÉRENCES BIBLIOGRAPHIQUES}

Barzin, Marcel, «L'esquisse d'une philosophie des valeurs d'Eugène Dupréel», in: Ch. Perelman, éd., Eugène Dupréel. L'homme et l'œuvre, Bruxelles, Editions de l'Institut de Sociologie, 1968, p. $26-40$. 
Bastide, Georges, «Le pluralisme axiologique d'Eugène Dupréel», in: Ch. Perelman, éd., Eugène Dupréel. L'homme et l'œuvre, Bruxelles, Editions de l'Institut de Sociologie, 1968, p. 41-59.

Berthelot, René, Evolutionnisme et platonisme, Paris, Alcan, 1908.

- Un romantisme utilitaire. Etude sur le mouvement pragmatiste, Paris, Alcan, vol. I, 1911, vol. II, 1913, vol. III, 1922.

Boudon, Raymond, Le juste et le vrai, Paris, Fayard, 1995.

Bouglé, Célestin, «Notice sur The Principles of Sociology par F. H. Giddings », L'Année sociologique, vol. I., 1896-1897.

Coenen-Huther, Jacques, «La sociologie de Tönnies, les formes de sociabilité et l'Ecole sociologique de Bruxelles », Recherches sociologiques, Louvain-la-Neuve, vol. XXVIII, n 1, 1997, p. 91-96.

- «Sens moral ou raisons fortes?», L'Année sociologique, vol. 51, n 1, 2001, p. 233-254.

- «Esprit sociologique et relativisme moral», Revue européenne des sciences sociales, t. XLI, $\mathrm{n}^{\circ} 126,2003$, p. 169-180.

- Sociologie des élites, Paris, Armand Colin, 2004.

Croissant, Jeanne, «Dupréel et Protagoras. Une interprétation conventionnaliste du relativisme protagoréen», in: Ch. Perelman, Ed., Eugène Dupréel. L'homme et l' œuvre, Bruxelles, Editions de l'Institut de Sociologie, 1968, p. 203-226.

Davy, Georges, «Compte rendu de Sociologie générale par E. Dupréel», L’Année sociologique, troisième série, 1948-1949, p. 123-130.

- «Le rapport social est-il le tout de la philosophie de Dupréel?», in: Ch. Perelman, éd., Eugène Dupréel. L'homme et l'œuvre, Bruxelles, Editions de l'Institut de Sociologie, 1968, p. 130-140.

Donati, Pierpaolo, «La relation comme objet spécifique de la sociologie», Revue du Mauss semestrielle, $\mathrm{n}^{\circ} 24,2004$, p. 233-254.

Dupréel, Eugène, Le rapport social, Paris, Alcan, 1912.

- «Convention et raison», Revue de Métaphysique et de Morale, t. XXXII, 1925. Repris dans Essais pluralistes, 1949, p. 1-26.

- «Le problème sociologique du rire», Revue philosophique, t. CVI, 1928a. Repris dans Essais pluralistes, 1949 , p. $27-69$.

- «De la nécessité», Archives de la Société Belge de Philosophie, fasc. 1, 1928b. Repris dans Essais pluralistes, 1949, p. 70-105.

- Traité de morale, 2 vol., Bruxelles, Editions de la Revue de l’Université, 1932.

- «La cause et l'intervalle ou ordre et probabilité», Archives de la Société Belge de Philosophie, fasc. 2, 1933. Repris dans Essais pluralistes, 1949, p. 196-235.

- «La probabilité ordinale», Recherches philosophiques, t. III, 1933-1934. Repris dans Essais pluralistes, 1949, p. 236-249.

- «Les deux racines de la valeur du vrai», Revue de l'Université, décembre-janvier 1935. Repris dans Essais pluralistes, 1949, p. 285-299.

- Esquisse d'une philosophie des valeurs, Paris, Alcan, 1939.

- Sociologie générale, Paris, Presses Universitaires de France, 1948.

- «La pensée confuse », Annales de l'Ecole des Hautes Etudes de Gand, t. III, 1939b. Repris dans Essais pluralistes, 1949, p. 324-334.

- Essais pluralistes, Paris, Presses Universitaires de France, 1949.

- Similitude et dépassement, Bruxelles et Paris, Université Libre de Bruxelles, Travaux de la Faculté de philosophie et lettres, Presses Universitaires de Bruxelles et Presses Universitaires de France, 1968.

Ferry, Luc et Vincent, Jean-Didier, Qu' est-ce que l' homme? Paris, Odile Jacob, 2000.

Génard, Jean-Louis, «A propos de l'Esquisse d'une philosophie des valeurs», Revue de l'Institut de Sociologie, n³-4,1995 (paru en 1998), p. 33-84.

Goriely, Georges, «Réflexions critiques d'un disciple», in: Ch. Perelman, éd., Eugène Dupréel. L'homme et l'œuvre, Bruxelles, Editions de l'Institut de Sociologie, 1968, p. 95-103. 
Gurvitch, Georges, Déterminismes sociaux et liberté humaine, Paris, Presses Universitaires de France, 1955.

Gurvitch, Georges, éd., Traité de sociologie, vol. I, Paris, Presses Universitaires de France (3éd.), 1967.

James, William, The Will to Believe and Other Essays in Popular Philosophy, New York, Longmans Greens, 1896.

Joas, Hans, Kreativität des Handelns, trad. fr. : La créativité de l'agir, Paris, Cerf, 1999.

Lestel, Dominique, Les origines animales de la culture, Paris, Flammarion, 2001.

Mead, George Herbert, Mind, Self and Society, Chicago et Londres, The University of Chicago Press, 1962 (1934).

Perelman, Chaïm, «A propos d'Eugène Dupréel. Contribution à un portrait philosophique », in: $\mathrm{Ch}$. Perelman, éd., Eugène Dupréel. L' homme et l'œuvre, Bruxelles, Editions de l'Institut de Sociologie, 1968, p. 227-237.

Perelman, Chaïm et Olbrechts-Tyteca, Lucie, Traité de l'argumentation. La nouvelle réthorique, Bruxelles, Editions de l'Institut de Sociologie, 1970.

Simmel, Georg, Soziologie, 1908, trad. fr. : Sociologie. Etudes sur les formes de la socialisation, Paris, PUF, 1992.

Wilson, James Q., The Moral Sense, New York, Macmillan/The Free Press, 1993. 